\title{
An ECM-PSO based optimal charging current pattern searching for Li-ion batteries
}

\author{
Haitao Min", a, Weiyi Sun", b, Zhiru Liü, c, Yuanbin Yu, d, Dongni Guo ${ }^{3, e}$ \\ ${ }^{1}$ State Key Laboratory of Automotive Simulation and Control, Jilin University, Changchun 130022, \\ PR China. \\ ${ }^{2}$ Battery System R\&D Institute, Shenzhen KLclear Technology Co., Ltd, Shenzhen 518000. \\ ${ }^{3}$ FAW Bus and Coach Co., Ltd. Changchun 130033, PR China. \\ a minht@jlu.edu.cn; bsunwy14@mails.jlu.edu.cn, cliuzhiru@klclear.com; \\ dyyb@jlu.edu.cn, e guodongni@rdc.faw.com.cn
}

Keywords: Li-ion batteries, ECM, PSO algorithm, Multistage constant current charging.

\begin{abstract}
EV battery charging is growing in importance as it has a direct influence on the EV performance. Multistage Constant Current (MCC) charging strategy is an effective rapid charging method. This paper proposes an ECM-PSO algorithm to search for the optimal current pattern of the MCC charging strategy. The MCC charging model is built according to the Li-ion battery equivalent circuit model, and the object function for the ECM-PSO optimization was formulated and the optimal current pattern of the MCC charging method was obtained through the ECM-PSO algorithm. The experimental result shows that the obtained current pattern is capable of charging the batteries to $87.7 \%$ of the rated capacity within $36.5 \mathrm{~min}$. Compared with the conventional CC-CV charging method, the proposed charging strategy has a performance improvement of $60.3 \%$ in charging time reduction, and the average temperature rise during the proposed MCC charging process is $0.5{ }^{\circ} \mathrm{C}$ lower than the CC-CV charging method.
\end{abstract}

\section{Introduction}

Electric vehicle (EV) has been the new development point of motor industry because of the advantages of zero pollution and high energy efficiency [1-3]. Li-ion batteries with high energy density, good cycle-life performance, and low self-discharge rate [4-7] are getting more widely used in electric vehicles (EVs). However, the Li-ion battery charging strategy has become the bottleneck of their application, due to the slow charging speed and uncertainty effects on battery life. Li-ion battery charging optimization technology has become one of the difficult hotspots [8].

In $[9,10]$ the advantages of the multistage constant current (MCC) charging strategy have been confirmed. As illustrated in Fig. 1, the MCC charging strategy is consisted with several constant current charging stages. The MCC charging scheme relaxes the chemical reaction stress and could get a shorter charge time, which makes it suitable for rapid charging. Many researches have been taken on MCC charging strategy. The effect of different numbers of current stages was tested in [11], and it is proved that the increasing in charging stages of more than 5 shows almost negligible improvement on the charging time, and the proper current strategy should be 5. In [12], a Taguchi-based algorithm was used to obtain rapid charge. In [13], an Ant Colony System algorithm was used to select the optimum charging current among five charging stages, and the charging time was decreased and battery cycle life was extended by $25 \%$. 


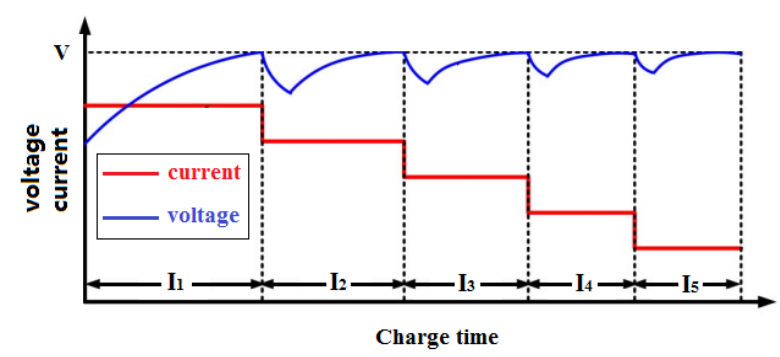

Fig. 1 MCC charging strategy.

This paper proposed an ECM-PSO current pattern searching method for the MCC charging strategy. The ECM (equivalent circuit model) of Li-ion battery was researched, the MCC charging model was built according to the ECM and the parameter of the MCC model was identified through battery pulse discharging experiment. The PSO method was applied to search for the optimal current pattern of the MCC charging method.

\section{ECM- PSO based optimal charging strategy search}

\subsection{Battery model.}

To optimize the charging current of the MCC charging procedure, an accurate and reliable battery model must be formulated when using model-based method. The ECM approach uses the circuit elements to represent the electrochemical phenomena inside the battery to avoid the lengthy electrochemical process calculation while still capturing the dynamic performance of the battery.

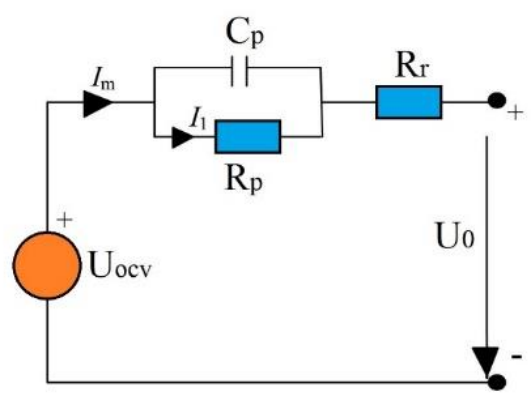

Fig. 2 One RC-branch ECM.

As shown in Fig. 2, there are four independent parameters in the model, namely $u_{o c v}, R_{r}, R_{p}$, and $C_{p \text {. }}$ $u_{\text {ocv }}$ is the open circuit voltage; $R_{r}$ is the ohmic resistance; $R_{p}$ is the polarization resistance and $C_{p}$ is the polarization capacity. The voltage on $R_{r}$ and $C_{p}$ is represented as $u_{p}$, the voltage on $R_{r}$ is $u_{r}$ and $I_{m}$ is the battery current.

From the circuit, the following formulations can be obtained:

$$
\begin{aligned}
& C_{p} \frac{d_{u p}(t)}{t}+\frac{u_{p}(t)}{R_{p}}=I_{m} \\
& u_{0}(t)=u_{\text {ocv }}+I_{m} \cdot R_{r}+u_{p}(t)
\end{aligned}
$$

\subsection{Object function.}

In order to attain the optimized design, a proper optimization problem must be formulated. Here, the main emphasis is to decrease the charging time and charge as much as possible capacity into the battery while improving the cycling life and energy transfer efficiency. To achieve this, the following objective function is defined:

Minimize

$$
\begin{aligned}
& \mathrm{S}=k_{1} \cdot C T+k_{2} \cdot T-k_{3} \cdot C P_{\text {norm }} \\
& C P_{\text {norm }}=\frac{C P}{C O} \\
& \left\{\begin{array}{c}
0 \mathrm{~min} \leq C T \leq 60 \mathrm{~min} \\
80 \% \leq C P_{\text {norm }} \leq 100 \% \\
T_{\text {amb }} \leq T \leq 40^{\circ} \mathrm{C} \\
0.01 \mathrm{C} \leq \mathrm{I} \leq 2.0 \mathrm{C} \\
I_{j} \leq I_{k} \text { if } j \leq k, j, k=1,2, \ldots, m
\end{array}\right.
\end{aligned}
$$


Where $C P_{\text {norm }}$ is the normalized charged capacity, which is defined as the percentage of the charged capacity to the rated capacity CQ; $k_{1}, k_{2}$, and $k_{3}$ is the weight factor of charging time $C T$, battery temperature $T$, normalized charged capacity $C P_{\text {norm }}$ respectively.

\subsection{ECM- PSO algorithm.}

As an emerging intelligent optimization algorithm, Particle Swarm Optimization (PSO) has been widely researched and applied to tackle optimization algorithm. Compared to other optimization algorithms, PSO algorithm is quite effective because only two model equations are required.

To get the optimized charging current pattern, the battery need to be charged with different current combinations. However, the number of all the possible combinations for different charging current pattern is very big and battery charging experiment is a time-consuming work, which makes it inefficient to test every possible current pattern.

To solve this problem, an ECM-PSO searching algorithm is adopted in this research. The algorithm used ECM to simulate the multistage constant current charging process of the battery and adopted the PSO algorithm to search for the optimal current pattern.

The flow chart of the ECM-PSO algorithm is summarized by the following steps.

1) Particle initialization. The initial charging patterns of each battery for stage 1 are randomly generated.

2) Battery charging simulation. Based on the ECM model, the charging process of each battery is simulated, and the charging time ${ }^{C T}$ and normalized discharge capacity $C P_{\text {norm }}$ is obtained.

3) Fitness function evaluation. For each particle, the fitness function is evaluated according to the charging time $C T$, battery temperature $\mathrm{T}$ and normalized discharge capacity $C P_{\text {norm }}$.

4) $P_{\text {best }}$ and $G_{\text {best }}$ calculation. For each particle, compare the fitness value with its $P_{\text {best }}$ value, if the current fitness value is better than $\mathrm{P}_{\text {best }}$, it becomes the new local best value. Identify the particle with the best $P_{\text {best }}$ value to get the global best value $G_{\text {best. }}$.

5) Convergence determination. Compare the average fitness value of current particle swarm with the previous particle swarm, if the difference is less than the threshold value $\alpha$, the search procedure is terminated.

6) Particle position update. If the termination condition is not achieved, update each particle's position with the data of $P_{\text {best }}$ and $\mathrm{G}_{\text {best }}$.

7) Repeat the steps from 2) to 6) until the best point is found or a fixed number of iterations has been reached.

\section{Results and discussion}

After 30 iterations, the difference of the average fitness value between before-and-after experiment is smaller than 0.005 , thus the specific termination conditions satisfied the default convergence criteria, and the PSO searching process was terminated. The optimal solution found by the searching algorithm after is $[2.00,1.02,0.78,0.61,0.42] \mathrm{C}$. The corresponding waveforms of the battery current and the simulated voltage according to this current pattern is plotted in Fig. 3.



Fig. 3 Proposed multistage charging strategy. 
To demonstrate the feasibility of the ECM-PSO algorithm previously discussed, a CC-CV charging of the battery was executed and the voltage and temperature of the battery during the charging process was compared with the charging process with the proposed charging pattern. Fig. 4 shows the comparison of the proposed optimal multistage $\mathrm{CC}$ charging strategy with $\mathrm{CC}-\mathrm{CV}$ charging strategy. From Fig. 4, it can be seen that the charging time of the proposed charging pattern required can greatly be improved although the charged capacity is lower than that of the $\mathrm{CC}-\mathrm{CV}$ method.

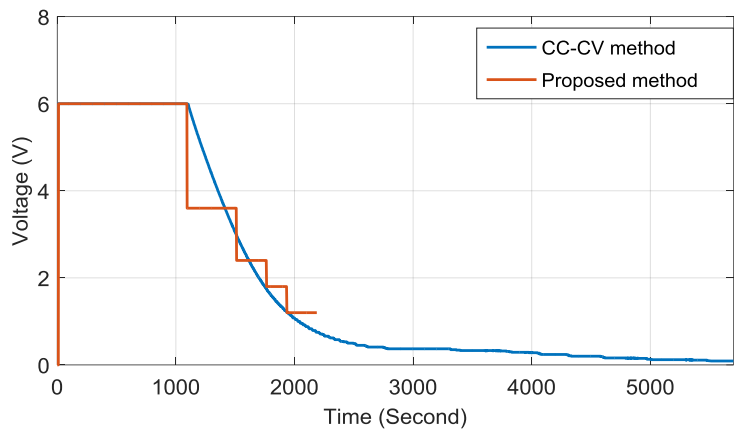

Fig. 4 Comparison of the proposed method and CC-CV method.

Table 1. lists the charging time, charged capacity and average temperature rise obtained using the proposed optimal charging strategy and $\mathrm{CC}-\mathrm{CV}$ strategy. It can be concluded from the experiment data that, the proposed strategy improved $60.3 \%$ of the charging time compared with the CC-CV strategy and the charged capacity of the proposed strategy is $87.7 \%$ of the CC-CV method and the average temperature rise during the proposed MCC charging process is $0.5^{\circ} \mathrm{C}$ lower than the CC-CV charging method. Therefore, the proposed MCC charging method is suitable for rapid charging application. Also, the lower temperature rise improves the charging efficiency and extends the battery life.

To verify the improvement of battery life, battery cycling tests were conducted. For the conventional CC-CV charging method, a $2 \mathrm{C}$ constant current is applied and for the proposed MCC charging, the obtained optimal current pattern $[2.00,1.02,0.78,0.61,0.42] \mathrm{C}$ is applied. The tests result shows that, the cycling number of the conventional CC-CV charging method is 246 when the capacity of the battery drops to $90 \%$ of the original capacity while the cycling number of the proposed MCC charging method is 298, which means the proposed MCC charging method extends $23 \%$ of the battery life compared to the conventional $\mathrm{CC}-\mathrm{CV}$ charging method.

Table 1. Comparison of the proposed charging method and the CC-CV method.

\begin{tabular}{cccc}
\hline & Propose method & CC-CV charging & Improvement \\
\hline Charging time $(\mathrm{min})$ & 36.5 & 92 & $60.3 \%$ \\
\hline Charged Capacity $(\mathrm{mAh})$ & 2569 & 2930 & $87.7 \%$ \\
\hline Average temperature rise $\left({ }^{\circ} \mathrm{C}\right)$ & 1.81 & 2.31 & 0.5 \\
\hline Cycling number to $90 \%$ capacity & 298 & 246 & $23 \%$ \\
\hline
\end{tabular}

\section{Conclusion}

This paper researched the optimal current pattern searching method for MCC charging strategy. The MCC charging model was built according to the ECM and the parameter of the MCC model was identified through battery pulse discharging experiment. The PSO method was applied to search for the optimal current pattern of the MCC charging method. An optimal charging current pattern has been found through the proposed algorithm and the experimental result shows that the obtained optimal charging pattern can charge the batteries to $87.7 \%$ of the rated capacity within $36.5 \mathrm{~min}$. A comparison with the CC-CV method is carried out. The experimental result shows that the proposed charging strategy has a performance improvement of $60.3 \%$ in charging time reduction, the average temperature rise during the proposed MCC charging process is $0.5{ }^{\circ} \mathrm{C}$ lower than the $\mathrm{CC}-\mathrm{CV}$ charging method and the proposed MCC charging method extends $23 \%$ of the battery life compared to the conventional CC-CV charging method. 


\section{Acknowledgements}

This research is sponsored by Key Project of Shenzhen Science \& Technology Innovation Committee, (No. 20150098) and Key Science and Technology Projects of Science and Technology Department of Jilin Province (20150204016GX).

\section{References}

[1]. X.G. Wu, C. Hu, J.Y. Du, and J.L. Sun, "Multistage CC-CV Charge Method for Li-Ion Battery." Mathematical Problems in Engineering, Article ID 294793, 2015.

[2]. Gan, Lingwen, Ufuk Topcu, and Steven H. Low. "Optimal decentralized protocol for electric vehicle charging." IEEE Transactions on Power Systems, vol. 28, no. 2, pp. 2013.

[3]. Pellegrino, Gianmario, et al. "Performance comparison between surface-mounted and interior PM motor drives for electric vehicle application." IEEE Transactions on Industrial Electronics, vol. 59, no. 2, pp. 803-811, 2013.

[4]. D. Ansean, M. Gonzalez, M. V. Garcia, C. J. Viera, C. J. Anton, and C. Blanco, "Evaluation of LiFePO4 batteries for electric vehicle applications," IEEE Transactions on Industry Applications, vol. 2, pp. 1855-1863, 2015.

[5]. H. Rahimi-Eichi, U. Ojha, F. Baronti, and M. Chow, "Battery management system: An overview of its application in the smart grid and electric vehicles," IEEE Ind. Electron. Mag., vol. 7, no. 2, pp. 4-16, Jun. 2013.

[6]. H. T. Lin, T. J. Liang, and S. M. Chen, "Estimation of battery state of health using probabilistic neural network,” IEEE Trans. Ind. Informat., vol. 9, no. 2, pp. 679-685, May 2013.

[7]. B. D. Valle, C. T. Wentz, and R. Sarpeshkar, "An area and power-efficient analog Li-ion battery charger circuit,” IEEE Trans. Biomed. Circuits Syst., vol. 5, no. 2, pp. 131-137, Apr. 2011.

[8]. H. Rahimi-Eichi, U. Ojha, F. Baronti, and M. Chow, "Evaluation of Acceptable Charging Current of Power Li-Ion Batteries Based on Polarization Characteristics," IEEE Ind. Electron., vol. 61, no. 12, pp. 6844-6851, Dec. 2014.

[9]. T. Ikeya et al., "Multi-step constant-current charging method for electric vehicle, valve-regulated, lead/acid batteries during night time for loadleveling," J. Power Sources, vol. 75, no. 1, pp. 101-107, Sep. 1998.

[10]. T. Ikeyaa et al., "Multi-step constant-current charging method for an electric vehicle nickel/metal hydride battery with high energy efficiency and long cycle life," J. Power Sources, vol. 105, no. 1, pp. 6-12, 2002.

[11]. L. R. Dung and J. H. Yen, "ILP-based algorithm for Lithium-ion battery charging profile," in Proc. IEEE Int. Symp. Ind. Electron., 2010, pp. 2286-2291.

[12]. Y.-H. Liu and Y.-F. Luo, "Search for an optimal rapid-charging pattern for Li-ion batteries using the Taguchi approach," IEEE Transactions on Industrial Electronics, vol. 57, no. 12, pp. 3963-3971, 2010.

[13]. Y.H. Liu, J.-H. Teng, and Y.-C. Lin, "Search for an optimal rapid charging pattern for lithium-ion batteries using Ant Colony System algorithm," IEEE Transactions on Industrial Electronics, vol. 52, no. 5, pp. 1328-1336, 2005. 